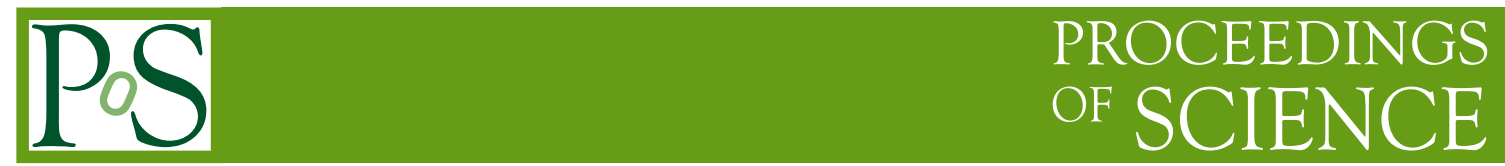

\title{
Overview of latest results from PHENIX
}

\section{Takao Sakaguchi for the PHENIX collaboration*}

Brookhaven National Laboratory

E-mail: takaodonl.gov

An overview of the latest results on the hard probes from the PHENIX experiment at RHIC is given. The results on the measurements of high $p_{T}$ hadrons, hadron-hadron correlations, open heavy flavor and quarkonia, and direct photons from large $(\mathrm{Au}+\mathrm{Au})$ to small collision systems $\left(p+\mathrm{Al}\right.$ and $\left.p / d /{ }^{3} \mathrm{He}+\mathrm{Au}\right)$ provided a deeper insight on the medium created in the large systems and the possible onset of QGP-nization in transition from small to large systems.

International Conference on Hard and Electromagnetic Probes of High-Energy Nuclear Collisions 30 September - 5 October 2018

Aix-Les-Bains, Savoie, France

${ }^{*}$ Speaker. 


\section{Introduction}

The Relativistic Heavy Ion Collider (RHIC) at Brookhaven National Laboratory (BNL) has been operated almost for two decades, during which variety of nucleus have been collided at various energies, in addition to the golden colliding mode of $\mathrm{Au}+\mathrm{Au}$ collisions at $\sqrt{s_{N N}}=200 \mathrm{GeV}$. In the last five years of data taking and analysis, PHENIX focused on not only large systems like $\mathrm{Au}+\mathrm{Au}$ collisions, but also small systems like $p+\mathrm{Al}$ and $p / d /{ }^{3} \mathrm{He}+\mathrm{Au}$ collisions. We will show the latest results from these systems and discuss what we learned.

\section{Results from large system}

From the beginning of the experiment, the measurement of the high $p_{T}$ hadrons has been of our primary focus. Figure 1 shows the latest compilation of the nuclear modification factors $\left(R_{\mathrm{AA}}\right)$ for various particles emitted in $0-10 \% \mathrm{Au}+\mathrm{Au}$ collisions at $\sqrt{s_{N N}}=200 \mathrm{GeV}$. It is clearly seen

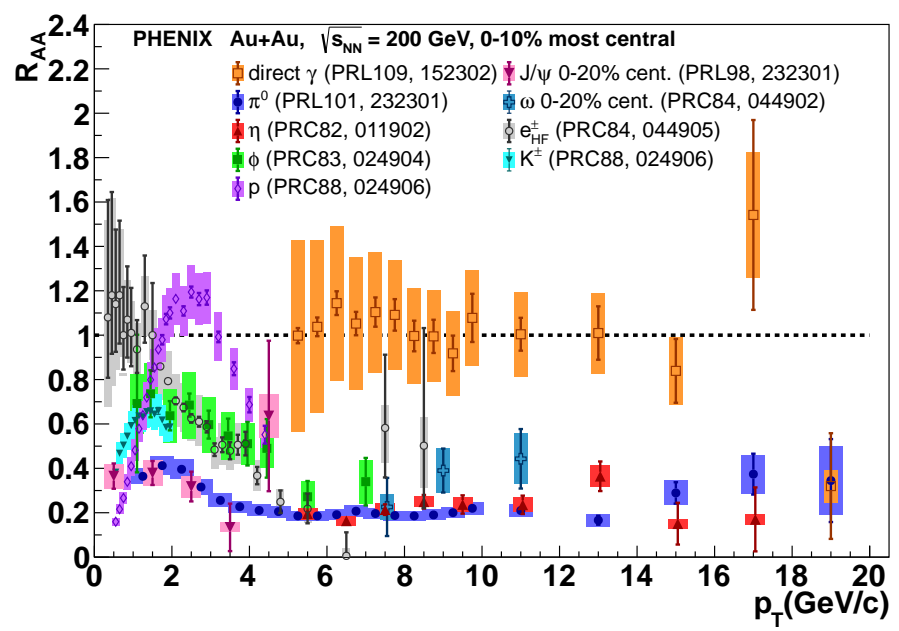

Figure 1: Latest compilation of $R_{\mathrm{AA}}$ for various particles in 0-10\% $\mathrm{Au}+\mathrm{Au}$ collisions at $\sqrt{s_{N N}}=200 \mathrm{GeV}$.

that the yields of light mesons are equally suppressed over $p_{T}$, except for $\phi$ at low $p_{T}$, while the direct photons are consistent with the expectation from the primordial production. With the $\mathrm{Cu}+\mathrm{Au}$ collisions performed in the RHIC Year-2012 run, we have extend the compilation to an asymmetric system, as shown in Fig. 2(a). The yields of light hadrons $\left(\pi^{0}, \eta, \omega\right)$ are again equally suppressed, while a strange hadron $(\phi)$ is off the trend in $p_{T}<5 \mathrm{GeV} / c$, which is consistent with the $\mathrm{Au}+\mathrm{Au}$ result. When looking at the integrated $R_{\mathrm{AA}}$ for $p_{T}>5-7 \mathrm{GeV} / c$ as a function of $N_{\text {part }}$ as shown in Fig. 2(b), the $R_{\mathrm{AA}}$ values follow a common trend. This is consistent with the fact that the $\phi$ is also equally suppressed when going to higher $p_{T}$ [1].

The hadron-hadron correlation gives us additional insight of the medium compared to single hadrons. PHENIX has measured the $\pi^{0}$-hadron correlations in Au+Au collisions at $\sqrt{s_{N N}}=200 \mathrm{GeV}$ in the past, and obtained the width of the near-side and away-side peak of jet functions [2]. At that time, the particle flow was explored up to second order $\left(v_{2}\right)$, therefore the background flow subtraction was performed only up to the second order as well. With the high statistics RHIC Year-2010 

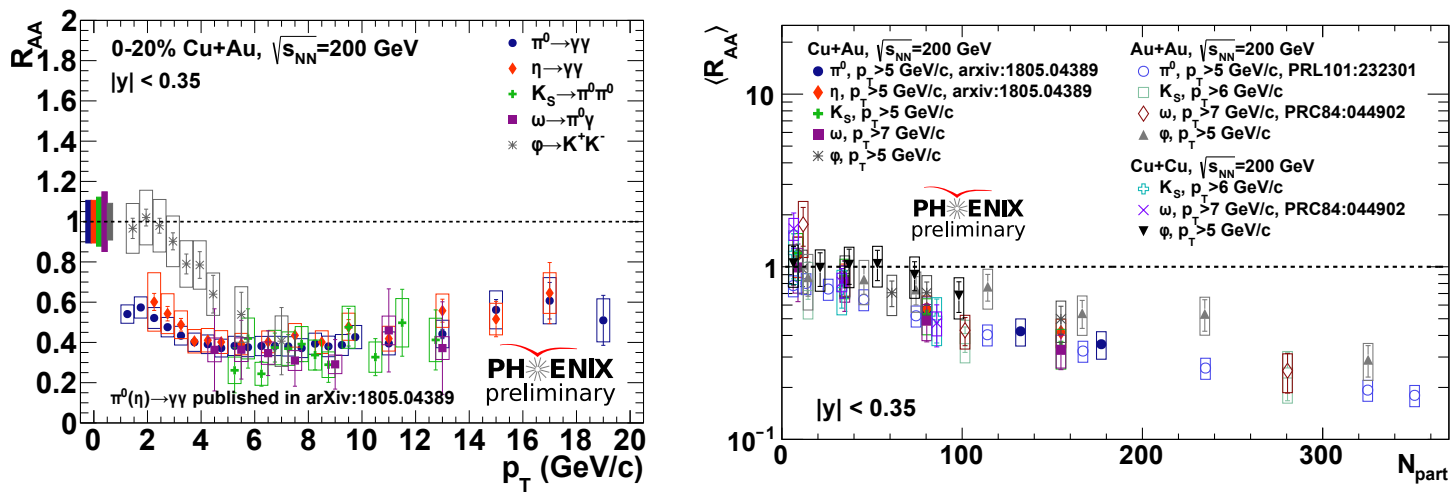

Figure 2: (a, left) $R_{\mathrm{AA}}$ for hadrons in $0-20 \% \mathrm{Cu}+\mathrm{Au}$ collisions at $\sqrt{s_{N N}}=200 \mathrm{GeV}$. (b, right) Integrated $R_{\mathrm{AA}}$ for $\mathrm{Au}+\mathrm{Au}, \mathrm{Cu}+\mathrm{Au}$ and $\mathrm{Cu}+\mathrm{Cu}$ collisions for $p_{T}$ above $5-7 \mathrm{GeV} / c$, as a function of $N_{\text {part }}$.

and 2011 run data and taking $v_{n}(n=2,3,4)$ flow components into account for background estimate, the jet functions are significantly improved and smooth in $\Delta \phi$ as shown in Fig. 3(a). The widths of the away-side peaks are shown in Fig. 3(b). Comparing to the previous result, both the statistical and systematic uncertainties are much improved, which results in a firmer conclusion that the widths are larger for $\mathrm{Au}+\mathrm{Au}$ collisions compared to that for $p+p$ at low $p_{T}$, and they converge as going to higher $p_{T}$ [3]. The result can be compared to the $\gamma$-hadron correlation result whose trigger particles don't interact with medium [4].
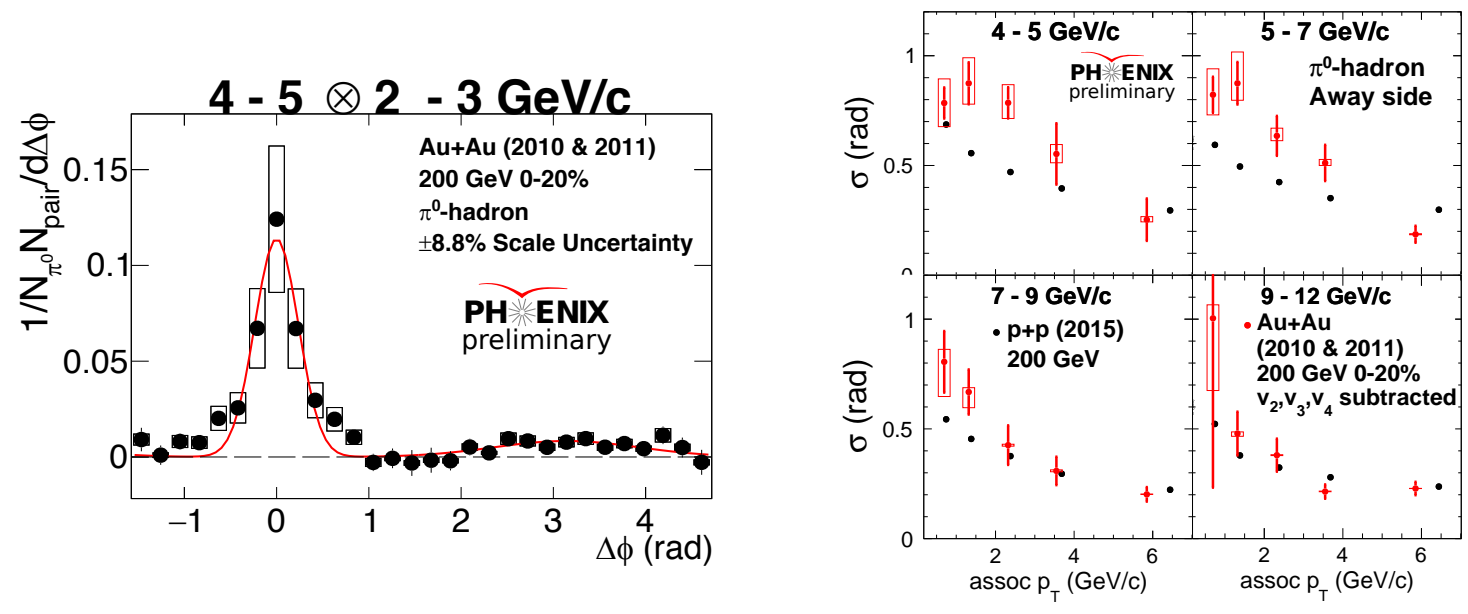

Figure 3: (a, left) Jet function from $\pi^{0}-h$ correlations in $0-20 \% \mathrm{Au}+\mathrm{Au}$ collisions at $\sqrt{s_{N N}}=200 \mathrm{GeV}$. (b, right) Away-side peak widths of the jet functions, as a function of trigger $\pi^{0} p_{T}$ and associated hadron $p_{T}$.

A systematic study of the energy loss as a function of quark mass gives another handle on the energy loss mechanism. PHENIX has measured electrons and muons from heavy flavor quark decay (charm and bottom) and unfolded to each component. Figure 4(a) shows the $R_{\mathrm{AA}}$ for the inclusive heavy flavor electrons, together with the electrons from charm and bottom separately in minimum bias $\mathrm{Au}+\mathrm{Au}$ collisions at $\sqrt{s_{N N}}=200 \mathrm{GeV}$. A hint of the mass ordering in the suppression is seen; electrons from bottom quarks tend to be less suppressed compared to those from charm quarks. The large errors, however, prevented us from making a definitive conclusion. A dominant 

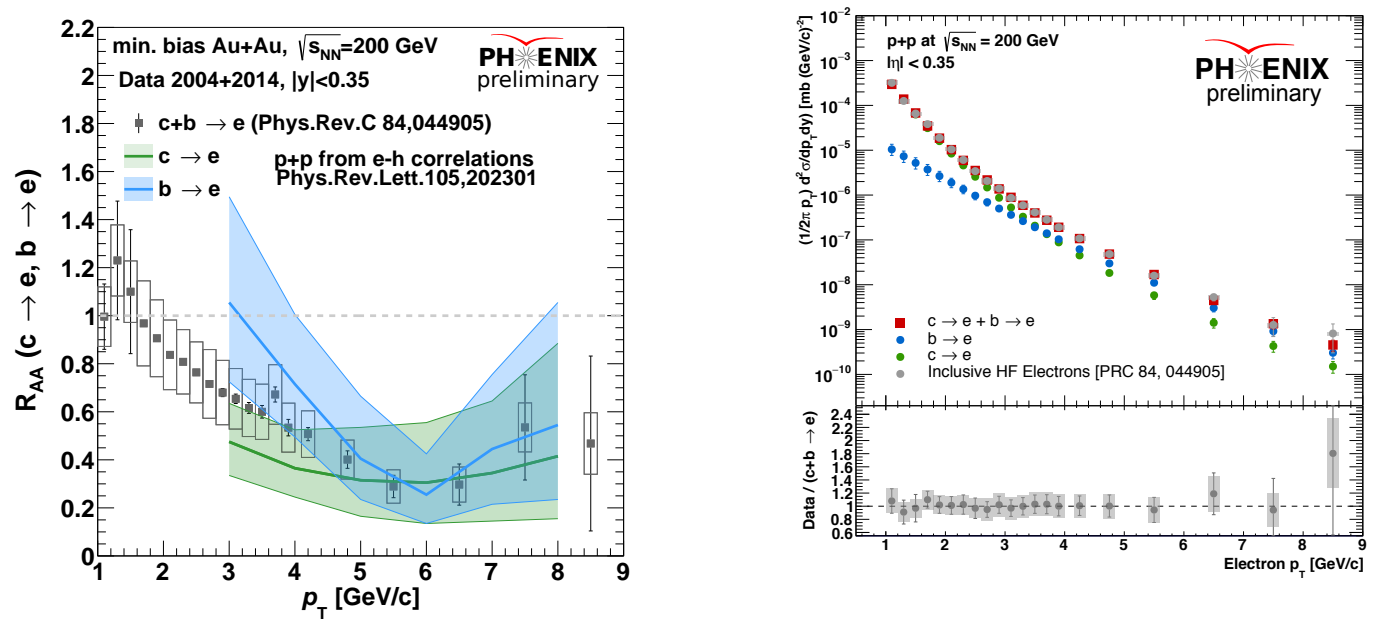

Figure 4: (a, left) $R_{\mathrm{AA}}$ for inclusive electrons from charm and bottom quarks, and electrons from charm and bottom quarks separately, measured in minimum bias Au+Au collisions at $\sqrt{s_{N N}}=200 \mathrm{GeV}$. (b, right) New $p+p$ baseline of the electrons from charm and bottom quarks.

source of uncertainty in this measurement is the fact that the $p+p$ reference was made up from the $e-h$ correlation result by the STAR experiment. With the RHIC Year-2015 data which has the VTX detector, PHENIX succeeded to measure the electrons from charm and bottom quarks separately in $p+p$ collisions, as shown in Fig. 4(b) [5]. A forthcoming $R_{\mathrm{AA}}$ measurement will use this new $p+p$ baseline. If the heavy quarks lose their energies significantly, they may eventually stop in the medium and follow the expansion of the bulk system, in which case these quarks will flow. PHENIX has also successfully measured the flow of electrons from charm and bottom quarks separately, as shown in Fig. 5. The electrons from bottom quarks seem to flow less than those from
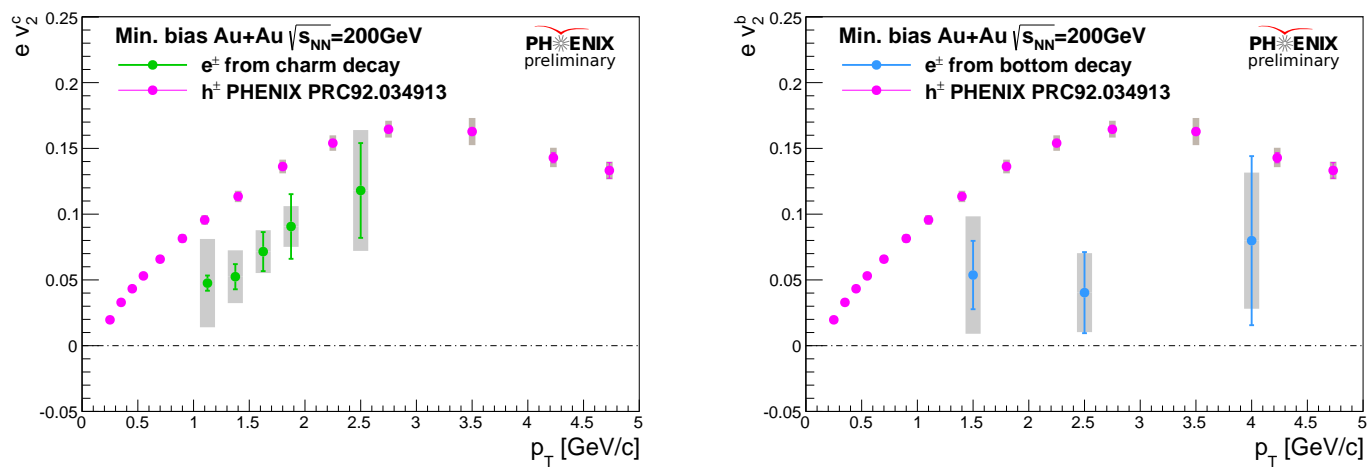

Figure 5: $v_{2}$ of the inclusive heavy flavor electrons compared with (a, left) unfolded charm electrons and (b, right) bottom electrons.

charm quarks. Together with $R_{\mathrm{AA}}$, the result implies that less energy loss of a heavy quark leads to less probability of the quark being stopped and merged into the bulk system [6]. 


\section{Transition from large to small systems}

Direct photons are a strong tool to shed a light on the thermodynamics of the systems, since the photons leave the system unscathed strongly once emitted. They are also useful for exploring the threshold of partonic matter production. PHENIX has studied low $p_{T}$ direct photon production for various energies and collision systems, and found intriguing $d N_{c h} / d \eta$ scaling. Figure 6 shows the direct photon spectra from large collision systems scaled by $\left(d N_{c h} / d \eta\right)^{1.25}[7,8]$. It shows that

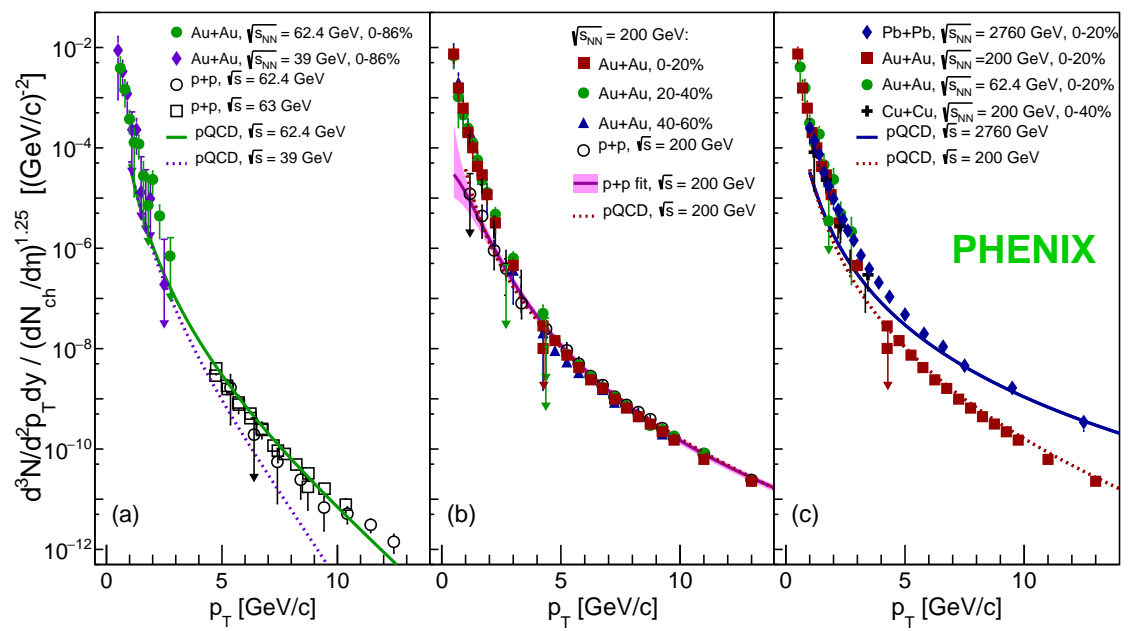

Figure 6: Direct photon invariant yields scaled by $\left(d N_{c h} / d \eta\right)^{1.25}$ for (a, left) Au+Au collisions at 39 and 62.4 GeV together with $p+p$ and pQCD calculation, (b, middle) Au+Au collisions at $200 \mathrm{GeV}$ for several centralities, and (c, right) central $\mathrm{Au}+\mathrm{Au}, \mathrm{Cu}+\mathrm{Cu}$ and $\mathrm{Pb}+\mathrm{Pb}$ collisions.

the scaled direct photon yield are lying on top of each other for $p_{T}<5 \mathrm{GeV} / c$ that are primarily soft photons emitted from the bulk system, irrespective of the collision systems, energies, or centralities.

PHENIX has also measured the direct photons in $p+\mathrm{Au}$ collisions at $\sqrt{s_{N N}}=200 \mathrm{GeV}$ as shown in Fig. 7. Although the errors are large, a hint of enhancement over the expectation from $p+p$ collisions is seen. The result is found to be consistent with hydrodynamic calculation within errors [9].

We have summarized the direct photon measurements from large to small systems in the form of integrated yield $\left(p_{T}>1 \mathrm{GeV} / c\right)$ as a function of $d N_{c h} / d \eta$ as shown in Fig. 8. The dotted line shows the fit to the $\mathrm{A}+\mathrm{A}$ data with a function of $d N_{\gamma} / d \eta=\beta\left(d N_{c h} / d \eta\right)^{\alpha}$, where $\alpha$ is fixed to 1.25. It is found that all the A+A points are on the dotted lines, while $p+p$ and $N_{\text {coll }}$ scaled pQCD calculations are on a different line which is parallel to the dotted line. The $p / d+\mathrm{Au}$ data points seem to fill the gap smoothly between $\mathrm{A}+\mathrm{A}$ and $p+p$ points, which suggests that the QGP-nization happens smoothly in that $d N_{c h} / d \eta$ range $[8,9]$.

\section{Results from small systems}

Since the discovery of collective flow of particles in central $p+\mathrm{A}$ collisions at RHIC and the LHC as well as $d /{ }^{3} \mathrm{He}+\mathrm{Au}$ collisions at RHIC, a question from the hard probe point of view has 

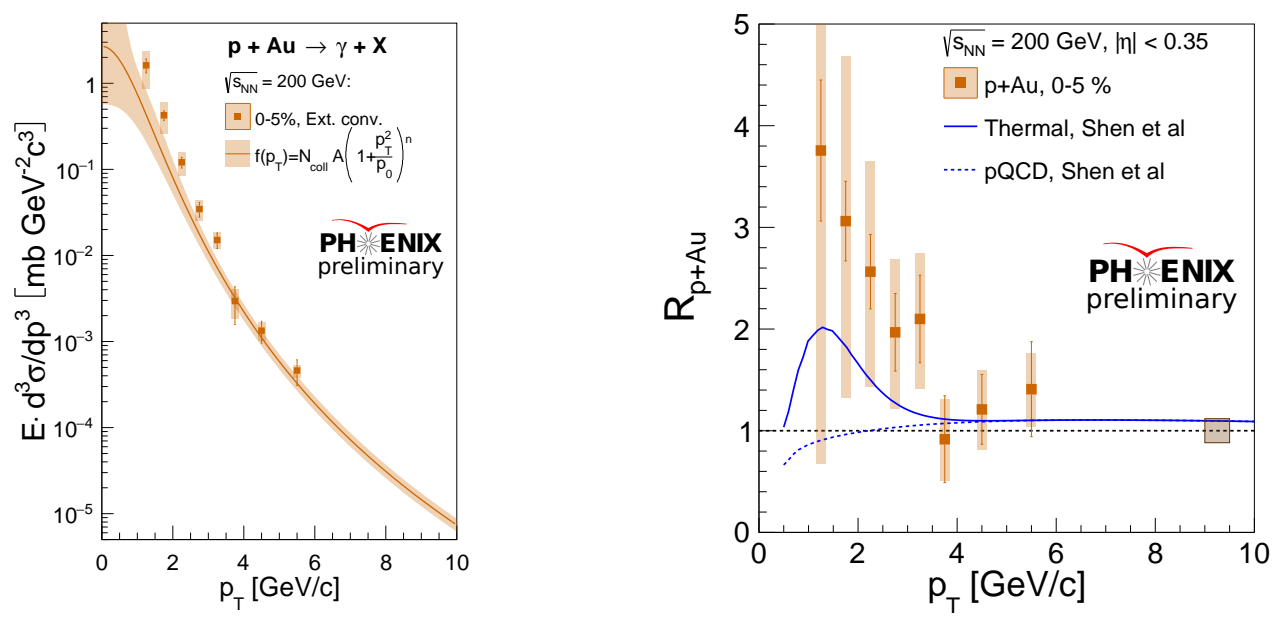

Figure 7: (a, left) Direct photon spectra in 0-5\% p+Au collisions at $\sqrt{s_{N N}}=200 \mathrm{GeV}$ together with the parameterized $p+p$ yield scaled by $N_{\text {coll }}$ ( (b, right) $R_{\mathrm{pA}}$ of the direct photons.

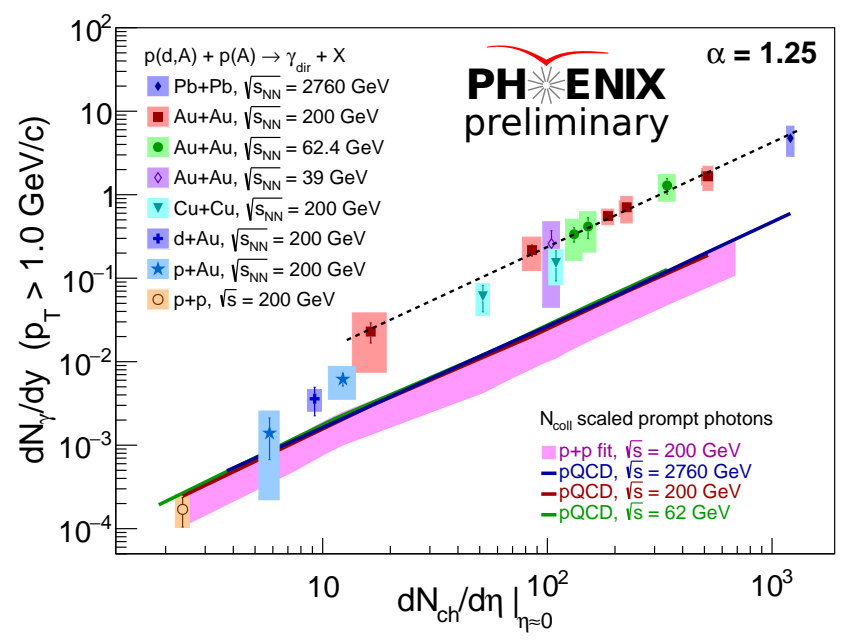

Figure 8: Integrated photon yield $\left(p_{T}>1 \mathrm{GeV} / c\right)$ as a function of $d N_{c h} / d \eta$ for various collision systems.

been whether or not the nuclear parton distribution function (nPDF) is strongly modified in these systems. PHENIX has measured muon-pairs at forward ( $p$-going) and backward (Au-going) rapidities in $p+\mathrm{Au}$ collisions at $\sqrt{s_{N N}}=200 \mathrm{GeV}$, and extracted the invariant mass and $p_{T}$ spectra for the Drell-Yan process, by subtracting the known hadron decay contribution, as shown in Fig. 9. The Drell-Yan process primarily probes the nPDF of the light quark sector. The $R_{\mathrm{pA}}$ shows that the data is well described by the PYTHIA event generator with the EPPS16 nPDF [10]. With the same dataset but a different kinematic cut, we have measured the bottom-quark pair cross-section in $p+\mathrm{Au}$ collisions as shown in Fig. 10. Although the errors are large, the agreement between data and PYTHIA+EPPS16 nPDF seems to be a bit worse, suggesting that the gluon part of nPDF has a room to improve since the bottom-quark pairs are primarily produced from gluons [10].

Charmonia provide another handle on nPDF. PHENIX has studied $J / \psi$ production in $p+\mathrm{Al}$ 

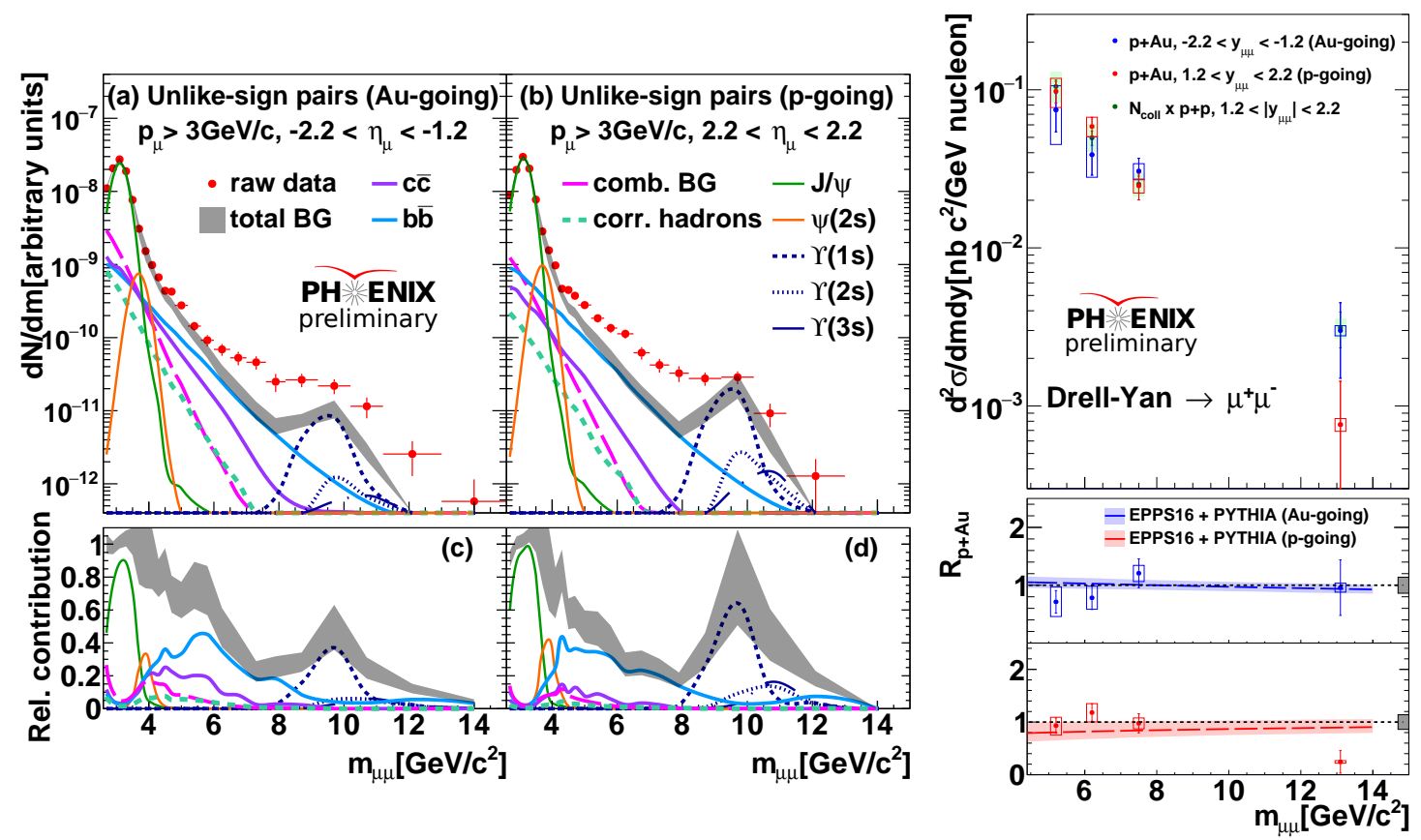

Figure 9: (a, left) Invariant mass spectra for muon-pairs in forward and backward rapidities in $p+$ Au collisions at $\sqrt{s_{N N}}=200 \mathrm{GeV}$, together with the various known hadron contributions. (b, right) $p_{T}$ spectra for the extracted Drell-Yan contribution for $p+p$ (scaled by $N_{\text {coll }}$ ) and $p+$ Au collisions, and corresponding $R_{\mathrm{pA}}$.

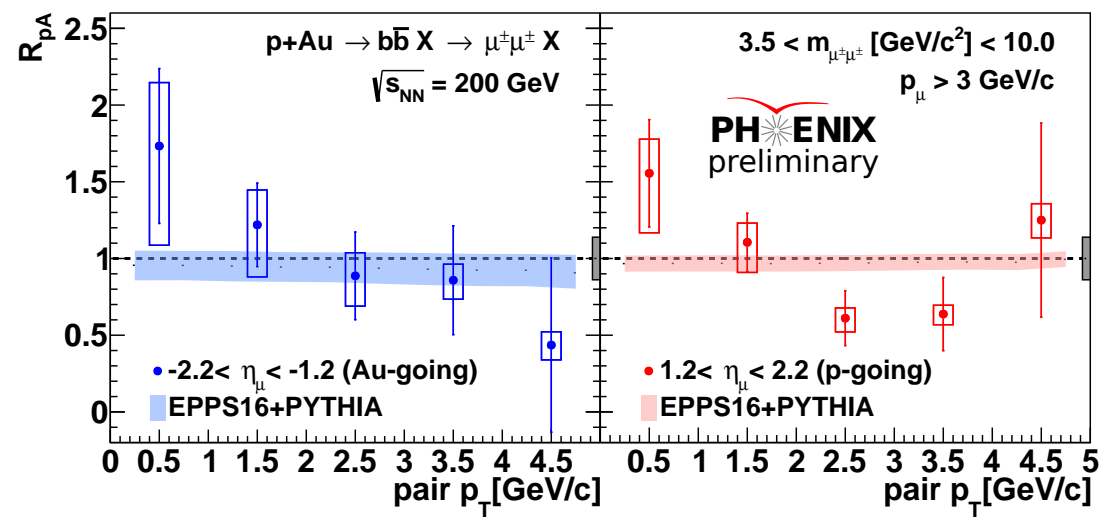

Figure 10: $R_{\mathrm{pA}}$ of the bottom-quark pairs at forward and backward rapidities.

and $p / d /{ }^{3} \mathrm{He}+\mathrm{Au}$ collisions at $\sqrt{s_{N N}}=200 \mathrm{GeV}$. Figure 11 shows the inclusive $J / \psi R_{\mathrm{AB}}$ at forward $\left(p / d /{ }^{3} \mathrm{He}\right.$-going) and backward (Au or Al-going) rapidities as a function of $N_{\text {part }}$. It is found that the $R_{\mathrm{AB}}$ scales very well with $N_{\text {part }}$ individually at forward and backward rapidities. In order to investigate differentially, we have performed the measurement of $J / \psi R_{\mathrm{AB}}$ as a function of $p_{T}$ as shown in Fig. 12. The $R_{\mathrm{AB}}$ for $p / d /{ }^{3} \mathrm{He}+\mathrm{Au}$ collisions are very consistent each other both at forward and backward rapidities, while that for $p+\mathrm{Al}$ collisions is out of trend, implying the $R_{\mathrm{AB}}$ is primarily determined by the nPDF or cold nuclear effects of the nucleus [11]. One thing worth noting is that the previous single muon measurement from heavy quarks shows a different trend at backward rapidities [12]; single muon $R_{\mathrm{dA}}$ is enhanced, while $J / \psi R_{\mathrm{AB}}$ is suppressed. This is 

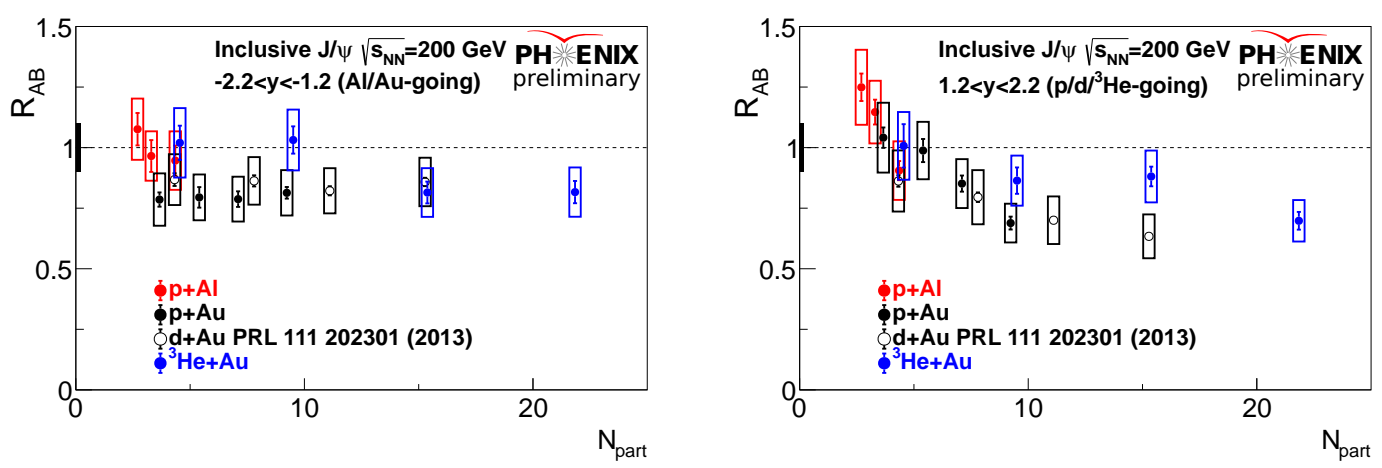

Figure 11: Inclusive $J / \psi R_{\mathrm{AB}}$ at forward at backward rapidities as a function of $N_{\text {part }}$.
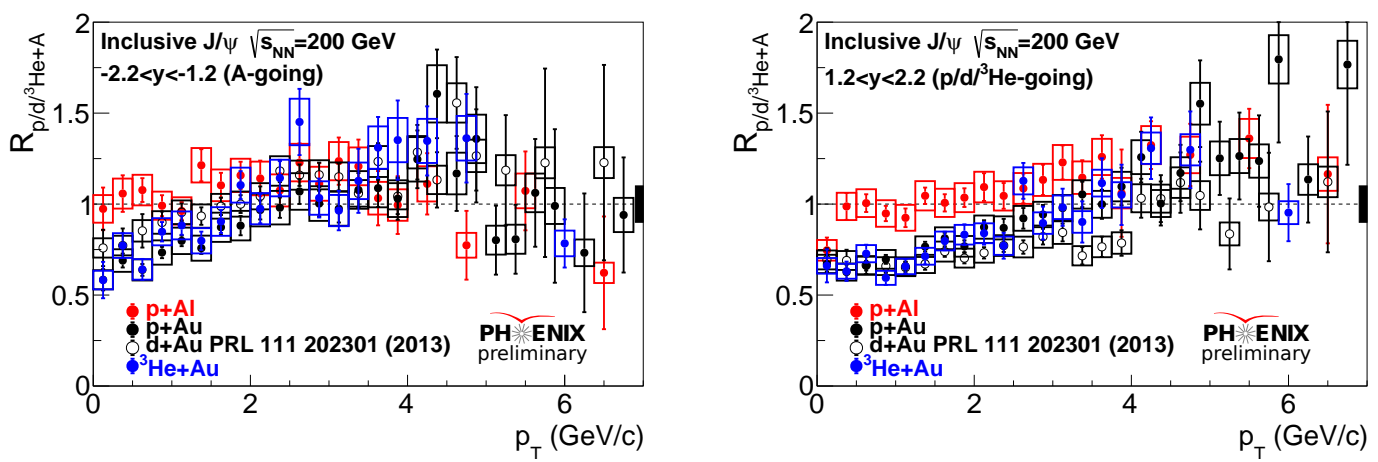

Figure 12: Inclusive $J / \psi R_{\mathrm{AB}}$ at forward at backward rapidities as a function of $p_{T}$.

consistent with the breakup of $J / \psi$ in the Au nucleus by the cold nuclear effects.

Lastly, PHENIX has recently published the collision energy and system size dependence of light hadron flow $\left(v_{2}\right.$ and $\left.v_{3}\right)$ in the small collision systems, and found that the results are well described by hydrodynamic calculations [13]. PHENIX has also measured the $v_{2}$ of muons from heavy quarks at forward ( $d$-going) and backward (Au-going) rapidities in most central $d+\mathrm{Au}$ collisions at $\sqrt{s_{N N}}=200 \mathrm{GeV}$ as shown in Fig. 13 [6]. Although the errors are large, it was found the

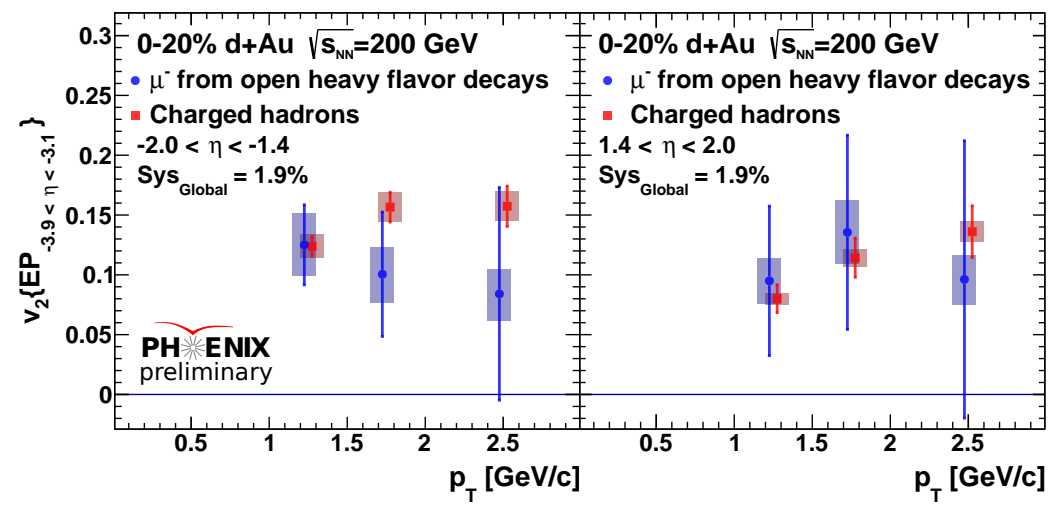

Figure 13: $v_{2}$ of heavy flavor muons and charged hadrons at forward and backward rapidities in $0-20 \%$ $d+\mathrm{Au}$ collisions. 
muons also flow and the magnitudes of the flow are consistent with those of charged hadrons. Taken together, these results are rather suggestive of QGP-droplet formation, which does not preclude the coexistence of conventional cold nuclear matter effects in these small systems.

\section{Summary}

We have presented the latest results on the hard probes from large to small collision systems by fully exploiting the flexibility of RHIC. The high $p_{T}$ hadrons in large systems are equally suppressed at the same $N_{\text {part }}$, except for a strange hadron, $\phi$. Away-side jet widths in Au+Au collisions are found to be larger than and consistent with those for $p+p$ collisions at low and high associated $p_{T}$, respectively. The electrons from charm quarks are found to flow more than those from bottom quarks. This is consistent with more energy loss for charm quarks. Soft photon yields $\left(p_{T}<5 \mathrm{GeV} / c\right)$ measured over various collision systems showed that the yields scale as: $d N_{\gamma} / d y=\alpha\left(d N_{c h} / d \eta\right)^{1.25}$. The photon yields in $p+$ Au collisions look to fill the gap between the yields in $p+p$ and $\mathrm{A}+\mathrm{A}$ systems when plotting against $d N_{c h} / d \eta$, hinting a transition from normal to partonic matter in this $d N_{c h} / d \eta$ region. Cold nuclear effects probed by Drell-Yan and bottom quarks are reasonably described by EPPS16 nPDF and PYTHIA event generator, modulo a bit worse description for bottom quarks, suggesting a room of improvement for gluon nPDF. Comparison of single muon and $J / \psi$ yields in small systems provided a strong proof of the breakup of $J / \psi$ in the Au nucleus. Both light-flavor hadrons and heavy quark muons are found to flow in central $d+$ Au collisions, implying that the "mini-QGP" production in small systems is rather suggestive.

\section{References}

[1] Y. Mitrankov, these proceedings.

[2] A. Adare et al., "Trends in Yield and Azimuthal Shape Modification in Dihadron Correlations in Relativistic Heavy Ion Collisions”, Phys. Rev. Lett. 104, 252301 (2010) [1002.1077 [nucl-ex]].

[3] M. Connors, these proceedings.

[4] A. Lebedev, these proceedings.

[5] T. Rinn, these proceedings.

[6] K. Nagashima, these proceedings.

[7] A. Adare et al., "Beam-energy and centrality dependence of direct-photon emission from ultra-relativistic heavy-ion collisions", [1805.04084 [hep-ex]].

[8] A. Drees, these proceedings.

[9] N. Novitzky, these proceedings.

[10] Y. Leung, these proceedings.

[11] M. Durham, these proceedings.

[12] A. Adare et al., "Cold-Nuclear-Matter Effects on Heavy-Quark Production at Forward and Backward Rapidity in $d+A$ u Collisions at $\sqrt{s_{N N}}=200 \mathrm{GeV}$ ”, Phys. Rev. Lett. 112, 252301 (2014) [1310.1005 [nucl-ex]].

[13] C. Aidala et al., "Creating small circular, elliptical, and triangular droplets of quark-gluon plasma", Nature Physics, doi:10.1038/s41567-018-0360-0 [1805.02973 [nucl-ex]]. 